www.jmscr.igmpublication.org

Impact Factor 5.244

Index Copernicus Value: 83.27

ISSN (e)-2347-176x ISSN (p) 2455-0450

crossref DOI: _https://dx.doi.org/10.18535/jmscr/v4i11.57

Journal Of Medical Science And Clinical Research

An Official Publication of IGM Publication

\title{
Factors Associated with the Use of Endoskeletal and Exoskeletal Prosthesis Among Lower Extremity Amputees - A Tertiary Care Centre Experience
}

\author{
Authors \\ Chitra $\mathbf{G}^{\mathbf{1}}$, Unnikrishnan $\mathbf{G}^{2^{*}}$, Meer Chisthi $\mathbf{M}^{3}$ \\ ${ }^{1}$ Assistant Professor, Department of Physical Medicine and Rehabilitation, Government Medical College, \\ Trivandrum, Kerala, India \\ ${ }^{2,3}$ Assistant Professor, Dept of General Surgery, Government Medical College, Trivandrum, Kerala, India. \\ Corresponding Author \\ Dr Unnikrishnan G
}

Department of General Surgery, Government Medical College, Trivandrum, Kerala, India 695011

Email:unni_ukg@rediffmail.com phone: 919961974443

\begin{abstract}
Background: Prostheses are used after amputation for better function, cosmetic appearance, and a psycho-spiritual sense of wellbeing. But they are mired by discomforts and disadvantages. Compared to the conventional exoskeletal prostheses, the modern endoskeletal ones claim to be functionally superior. The primary objective of the study was to compare the factors associated with the daily use of endoskeletal and exoskeletal prosthesis among lower extremity amputees who have used the prostheses for a minimum period of 3 months. Secondary objective was to compare the mobility status of persons using endoskeletal and exoskeletal prostheses and to find the difference in social and emotional aspects of persons using endoskeletal and exoskeletal prostheses.

Methods: This was a Cross sectional study, conducted from January 2014 to December 2014, at Government Medical College Hospital, Trivandrum. All the lower limb unilateral amputees who have used the prosthesis for a minimum period of 3 months who attended the Out Patient department of Physical Medicine and Rehabilitation Department were included in the study.

Results: The endoskeletal prostheses were more silent during ambulation, with a statistically significant difference. With regard to utility, it was found that exoskeletal prosthesis had better performance. Regarding ambulation, appearance, frustration, perceived response, residual limb health, social burden and well being no statistically significant difference was noted between the two types of prosthesis. Conclusions: Endoskeletal prosthesis and exoskeletal prosthesis both are having an almost equal acceptability among lower limb amputees. Exoskeletal prosthesis has better performance altogether while endoskeletal prosthesis is better in the silent ambulation aspect.

Keywords: functional outcome; lower extremity amputation; prostheses; quality of life.
\end{abstract}

\section{Introduction}

Normal human locomotion is achieved by a complex mechanism of precise alignment of lower limb skeletal system, joint motion and coordinated muscle activity, which gives a smooth forward progression. Therefore loss of lower limb gives rise to problems of body support, balance as well as of locomotion. 
Rehabilitation of disabled is one of the great challenges faced by governments all over world. On a rough estimate there are about 600 million orthopedically handicapped in the world out of which about 100 million are from India. A vast majority of the handicapped of the developing world are below poverty line. These disabled cannot afford the best form of artificial aids which are very costly and imported.

The history of amputation surgery and prosthetics begins right from the dawn of human medical thought. Its historical twists and turns parallel the development of medical science, culture, and human civilization itself. Prosthetics were developed with humankind's spiritual and functional need for wholesomeness. Prostheses catered for function, cosmetic appearance, and sometimes a psycho-spiritual sense of wholeness, but not necessarily in that order. Early prosthetic principles that were developed centuries ago exist to this day and are amazingly efficient in function.

The word Prosthesis, the proper name for an artificial limb derives from the Greek word which means "to place an addition". The prostheses of ancient cultures began as simple crutches or wooden and leather cups depicted in pottery. This grew into a type of modified crutch or peg to free the hands for functioning. An open socket peg leg had cloth rags to soften the distal tibia and fibula and allow a wide range of motion. These prostheses were very functional and incorporated many basic prosthetic principles. The greatest contribution to amputation surgery and prosthetics sciences of this time is by Ambroise Pare (1510 - 1590), a French army barber-surgeon. He reintroduced the use of linen ligatures originally put forth by Celsus and Hippocrates. Pare also invented upper and lower extremity prostheses that show knowledge of basic prosthetic function. He also invented an above knee prosthesis that was a kneeling peg leg and foot prosthesis. It had a fixed equines position, adjustable harness, knee lock control, and other engineering features used today.

In prosthetic management at the transtibial (Below Knee) level, there is general acceptance of biomechanical analysis of Radcliffe and almost all such amputees are fitted with "Patellar Tendon Bearing" prostheses of one design or another .' The total contact "Patellar Tendon Bearing" (PTB) socket is characterized by a bar in anterior wall designed to apply pressure to the patellar tendon. Pressure tolerant areas on below knee stump include the patellar tendon, the pretibial muscles, the gastronemius - soleus muscle the popliteal fossa, the lateral flat aspect of fibula and medial tibial flare. Pressure sensitive areas include the tibial crest, tubercle and condyle, the fibular head, the distal tibia and fibula, and the hamstring muscle. Despite the name "Patellar Tendon Bearing", the pressure should be equally distributed over pressure tolerant area and relieved over the pressure sensitive area. $^{2}$ While fabricating prostheses following principles are kept in mind: a) Only most important functions are duplicated. b) A reasonable and practical compromise is made between weight and strength of the prostheses. c) Amputee's gadget tolerance is not exceeded. d) Amputee's sound limb is not limited in any way. ${ }^{3}$ The primary goal is to produce a prosthesis, which permits unhindered function of the knee on the amputated side. To achieve this a total contact socket, which helps in distribution of weight and control forces generated between stump and socket when amputee walks, is required. ${ }^{4}$ In Endo-Skeletal prostheses weight is transmitted through a central shank from socket to foot and to ground. Therefore a central carbon fibre tube was designed, which could be attached to the socket above through a stainless steel upper coupling and lower coupling attaches it to foot. The upper coupling had a socket adopter with pyramid, which had provision to change the alignment of prostheses. Similarly lower coupling had SACH foot adopter with screws for alignment at lower level. ${ }^{8}$

Till recently, Below Knee amputee patients of 
armed forces used to be provided with conventional (Exoskeletal) prosthesis made of wood or plastic. In Exo-Skeletal Prosthesis, walls of prosthesis provide shape to the limb and also perform the weight bearing function. These prostheses usually are heavy and cumbersome. Keeping these facts in mind Artificial Limb Centre, Pune took up a project to develop state of art Below Knee Endo-Skeletal Prosthesis. In Endo-Skeletal prostheses a tube frame construction provides the weight bearing function. A flexible cosmetic foam cover provides the outer shape. As body weight is transmitted through a central shank, it is biomechanically and functionally better than Exo-Skeletal prostheses.

The first endoskeletal designs were used as temporary prostheses. In the first 10 years or so of the research program, the use of prostheses was discouraged because it was believed that more harm than good would result from the use of crudely made, poorly fitting sockets mounted on peg leg. Later after the rational for socket configuration was fully developed and plastic was proved useful in prosthetics with less time for fabrication revived the idea of temporary prosthesis. Pylons (endoskeletal Prostheses) with adjustable features began to appear in 1960. Their use was then accelerated by immediate post surgical fitting studies. These designs usually referred to as modular endoskeletal limbs have, gradually had more and more success despite the difficulty in shaping and maintaining their foam covering.

With this background, this study was undertaken to find the factors associated with the use of endoskeletal and exoskeletal prosthesis among lower extremity amputees. The primary objective of the study was to compare the factors associated with the daily use of endoskeletal and exoskeletal prosthesis among lower extremity amputees who have used the prostheses for a minimum period of 3 months. Secondary objective was to compare the mobility status of persons using endoskeletal and exoskeletal prostheses and to find the difference in social and emotional aspects of persons using endoskeletal and exoskeletal prostheses.

\section{Methods}

This was a Cross sectional study, conducted from January 2014 to December 2014, at Government Medical College Hospital, Trivandrum. The protocol was approved by the Institutional Review Board. All the lower limb unilateral amputees who have used the prosthesis for a minimum period of 3 months who attended the Out Patient department of Physical Medicine and Rehabilitation department of the hospital, were included in the study. After informed consent, all patients were recruited into the study.

Inclusion Criteria: Unilateral lower limb amputees who have used the prosthesis for a minimum period of 3 months.

Exclusion Criteria: Bilateral lower limb amputees, patients with severe cardiopulmonary disease, mental illness and cognitive defects, severe contralateral limb problems, scoliosis, bilateral visual impairment.

Base line information like age, address, income side of amputation, level of amputation, type of prosthesis used were collected using a pretested questionnaire. Mobility status, appearance, frustration, perceived response, residual limb health, social burden, sounds while walking, utility and well being were assessed by using a validated self administered Prosthesis Evaluation Questionnaire (PEQ) consisting of 82 questions developed by the Prosthesis Research Study, Seattle, WA, USA. The PEQ is divided into nine scales computed from 42 of the questions. These scales include ambulation, appearance, frustration, perceived response, residual limb health, social burden, sounds, utility and well being. The 40 remaining items pertain to other evaluation areas and are not grouped into scales. Individual questions of the PEQ are answered with respect to the amputees recollection of the previous 4 weeks. 


\section{Study Variables}

- Mobility status - ability to move around

- Social and emotional aspects - how frequently they are frustrated with their prosthesis, how their partner has responded to the prosthesis.

- Sound - mechanical sounds produced by prosthesis during ambulation

- Perceived response - desire to avoid strangers, whether handicap has affected their marital life etc.

- Well being - all round quality of life

- Residual limb health - how much they sweat inside the prosthesis, how smelly is the prosthesis etc.

Most questions in the PEQ use a visual analog scale format. Each visual analog scale is scored as a continuous numerical variable measured as the distance in millimetres from the left endpoint of the line to the point at which the respondent's mark crosses the line. Each time is $100 \mathrm{~mm}$ long and is always measured from the left (0-100). The questions are all worded so that a higher number (toward the right) will correspond with a more positive response. Descriptive analysis was done to find out percentages. Comparison was made using chi-square test \& T-test. All relevant data is presented as mean \pm standard deviation. A $p$ value of less than 0.05 was considered to be statistically significant.

\section{Results}

This is a comparison study of 33 candidates using endoskeletal prosthesis and 32 candidates using exoskeletal prosthesis. The study is carried out over a period of 1 year in the Department of Physical Medicine and Rehabilitations. All the lower limb unilateral amputees who have used the prosthesis for a minimum period of 3 months were included in the study.

In this study majority of the population among endoskeletal $36.36 \%$ and exoskeletal $43.75 \%$ come under the age group 41-55 yrs. The study showed a male preponderance: endoskeletal $90.9 \%$ and exoskeletal $84.38 \%$. Most of the candidates belonged to low socioeconomic group. Most common cause of amputation was road traffic accidents: endoskeletal $45.45 \%$ and exoskeletal $50 \%$. Next common cause was diabetes mellitus: endoskeletal $27.27 \%$ and exoskeletal 25\%. [Table 1] Endoskeletal prostheses dominated among patients with above knee amputation while there was equal distribution of exoskeletal and endoskeletal prostheses in below knee amputees. [Table 2]

Ambulation was assessed by rating the ability to walk up stairs, walk down stairs, walk in close spaces, ability to walk up a steep hill, walk down a steep hill etc. Both the groups showed good performance. There was no statistically significant difference: $\mathrm{p}$ value of 0.912 . Regarding the appearance of the prosthesis it was analyzed by rating how their prosthesis looked, damage done to their clothes by their prosthesis, damage done to their prosthesis cover etc. There was no statistically significant difference: $p$ value of 0.599 .

With regard to frustration with their prosthesis, both the group never had any kind of frustrations. They were happy with their prosthesis. Statistically there was no significant difference: $p$ value of 0.140 . Perceived response was rated by how their partner responded to their prosthesis and by rating whether their handicap affected their married life etc. Statistically no significant difference obtained between these two prosthesis.

Residual limb health was assessed by rating how much they sweat inside the prosthesis and by rating how smelly was their prosthesis etc. This was not statistically significant: $\mathrm{p}$ value of 0.69 . In our study regarding social burden and well being when assessed among persons using endoskeletal and exoskeletal prosthesis. There was no statistically significant difference. Most of person in both groups attended marriages and all other social functions. Well being when assessed by their quality of life found that majority of them were happy.

Mechanical sounds produced by the two 


\section{JMSCR Vol||04||Issue||11||Page 13851-13858||November}

prosthesis when assessed by rating how often their prosthesis made squeaking or clicking sounds and also by the rating how bothersome those sounds were. This was statistically significant: $\mathrm{p}$ value of 0.002. Endoskeletal prosthesis were found to be superior in this aspect. Utility of endoskeletal and exoskeletal prosthesis was compared and it was found that exoskeletal prosthesis had better performance. There is significant difference statistically: $p$ value of 0.039 .

Table 1. Reasons for amputations in lower limb amputees in the study

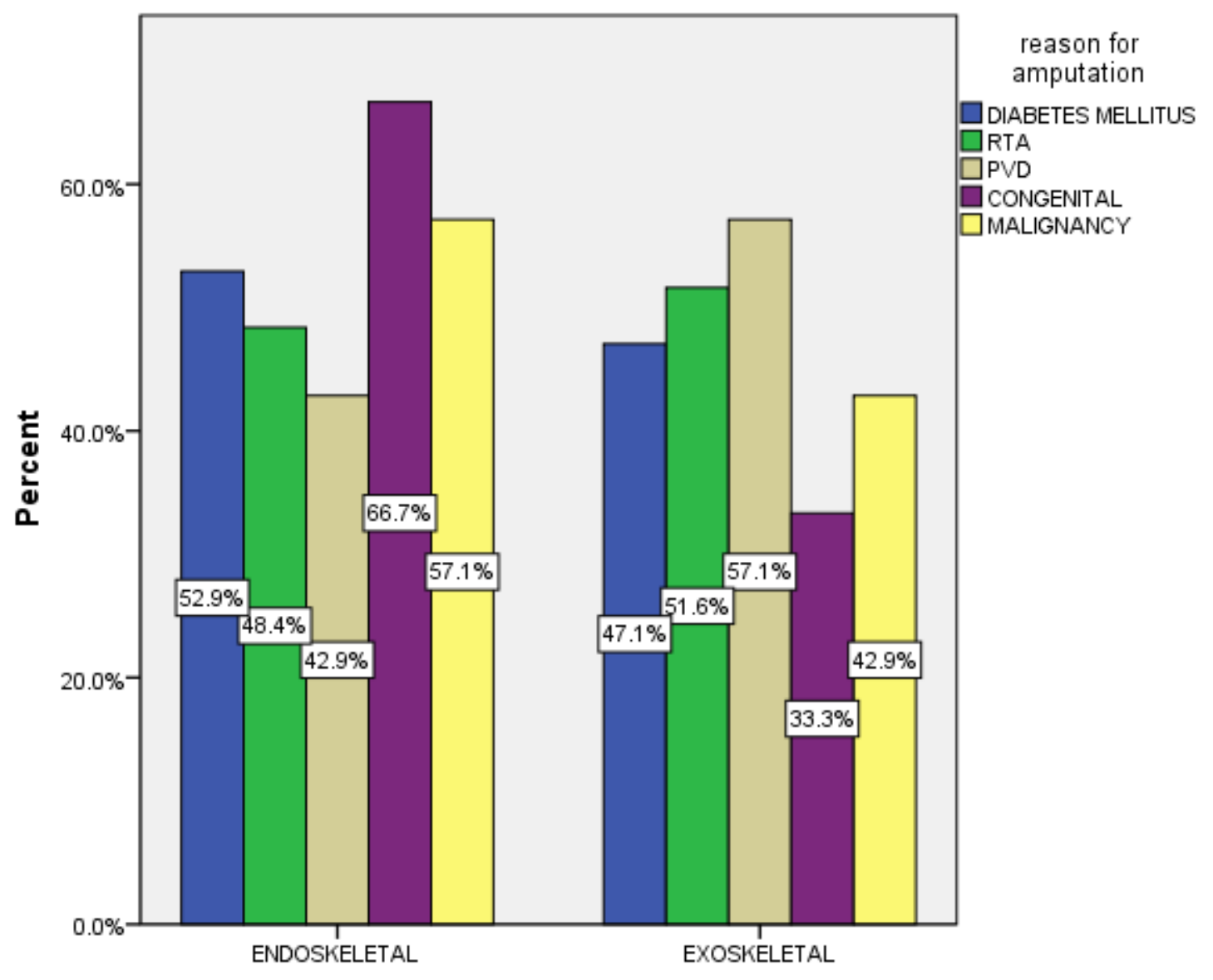

Type of prosthesis 
Table 2. Level of amputations in lower limb amputees in the study

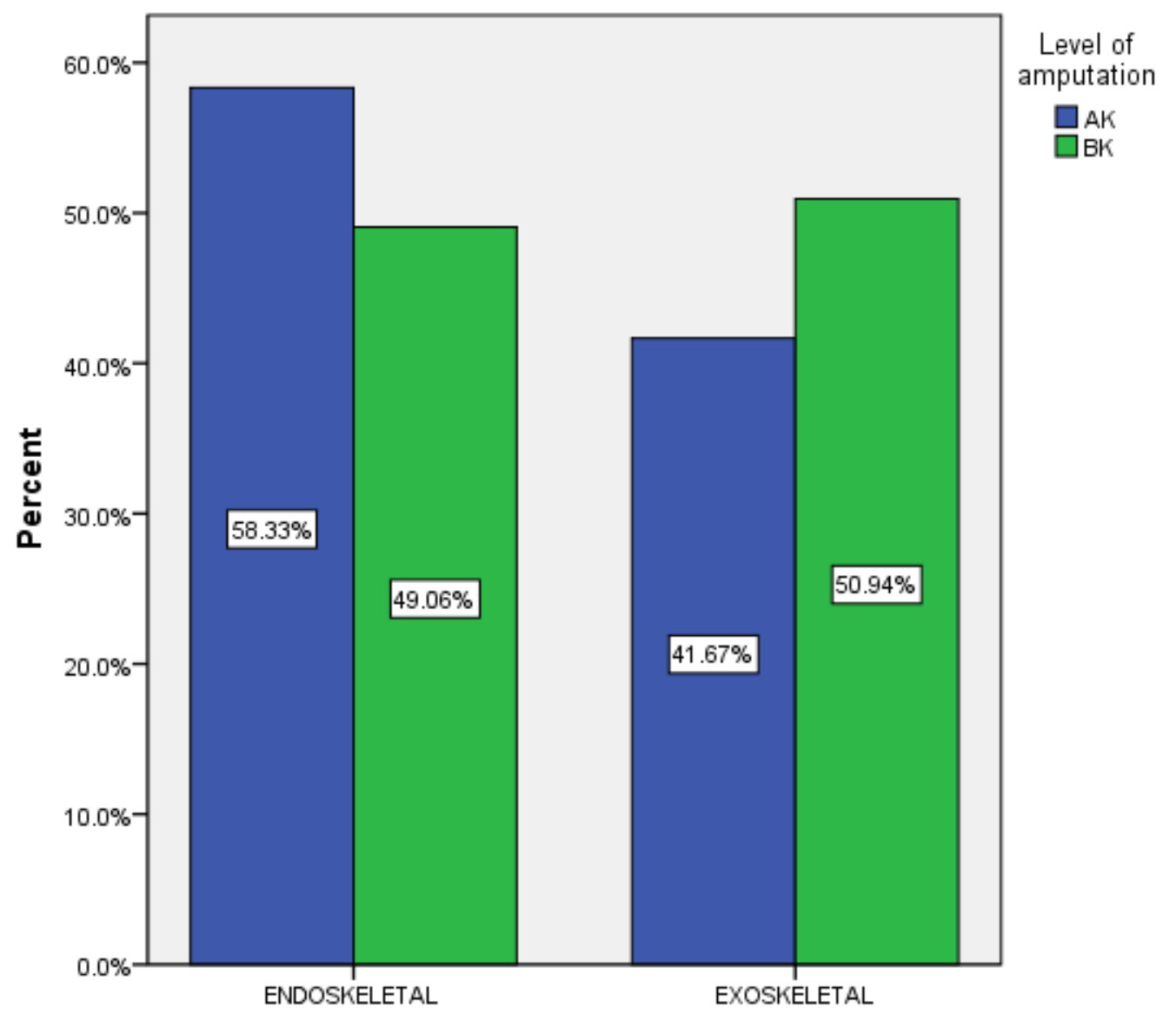

Type of prosthesis

\section{Discussion and Conclusions}

Lower limb amputees are dependent upon prostheses to achieve mobility. Therefore proper prosthetic fitting and suitable prosthetic components are necessary to optimise their performance in various activities. The design must anticipate the activity of the patient and the working environment to provide the greatest function. Rapid advances are taking place in the field of rehabilitation to improve the quality of life of disabled patients. Fabrication of artificial limb has also witnessed revolutionary changes in recent past.

Dianna Zidarov and her colleagues from the School of Rehabiitation, University of Montreal, Canada studied the quality of life with lower-limb amputation during rehabilitation and at 3-month follow-up. ${ }^{6}$ PEQ was used to study the quality of life. The mean prosthesis satisfaction scores were also high, indicating greater satisfaction with the prosthesis at discharge and at follow-up, and these scores did not differ significantly.

Laura L. Willingham et al from the Prosthetics Research Study, Seattle, WA, studied the function, performance, preference of transfemoral amputees transition from mechanical to microprocessor control of the prosthetic knee. ${ }^{7}$ Outcome measures was assessed using prosthesis evaluation questionnaire score. Response to PEQ showed a trend of increased satisfaction in the microprocessor control knee. This result was statistically significant $(\mathrm{P}<.001)$ between the microprocessor and the mechanical control.

Ruud W. Selles and his group from the Department of Rehabilitation Medicine, Erasmus MC Rotterdam, the Netherlands carried out a randomized controlled trial comparing functional outcome and cost efficiency of a total surfacebearing socket versus a conventional patellar tendon-bearing socket in transtibial amputees. ${ }^{8}$ 
Outcome measures was assessed using (PEQ score) prosthetic evaluation questionnaire score. In both groups, on average, changes in the PEQ scores were relatively small between baseline and follow-up and none of these changes were statistically significant.

In a study conducted by PK Gupta et al at the Armed Forces Medical College in India, it was found that various demographic factors, severity or duration of the disability or the level of rehabilitation do not influence patient satisfaction. ${ }^{9}$

Patients expressed more concern with aspects such as delay in issue of the prosthesis.

A comparative study of laminated exoskeletal and modular endoskeletal below knee prostheses was conducted at the Swami Vivekananda National Institute of Rehabilitation Training and Research (SVNIRTAR), Cuttack by Jagannatha Sahoo and his associates. ${ }^{10}$ They found that modular endoskeletal below knee prosthesis was superior to the exoskeletal laminated below knee prosthesis in terms of ease of fabrication, shorter hospital stay and being less expensive. It was also found that the endoskeletal modular below knee prosthesis was functionally better accepted than the exoskeletal laminated below knee prosthesis.

To conclude, our study shows that exoskeletal and endoskeletal prosthesis are almost equally efficient, with minor differences in some if the functionality and performance. However, our study had its own limitations in that the study was conducted in a relatively small sample size of 65 patients for a short period of only one year. Also, since the questionnaire was adapted from a western context, it would have had limitations in our set of patients.

Nevertheless, we were able to find statistically significant results to prove that both endoskeletal prosthesis and exoskeletal prosthesis are having an almost equal acceptability among lower limb amputees. The differences lie in that exoskeletal prosthesis provides better performance altogether while endoskeletal prosthesis scores well in the noise generated during ambulation aspect. Provision of prostheses to amputees is aimed at making their gait as normal as possible in terms of function, cosmesis and energy consumption. Irrespective of whether it is exoskeletal or endoskeletal, a properly fitted prostheses can aid the patient a lot in achieving near normal quality of life.

Acknowledgements: None.

Funding: None.

Conflict of interest: None declared.

Ethical approval: Obtained.

\section{References}

1. Colwell MO, Spires MC, Wontorcik, Davis AJ, Cauley P. Lower-Extremity. Prosthetics and rehabilitation. In: Grabois M, Garrison SJ, Hart KA, Lehmkuhl LD, editors. Physical Medicine and rehabilitation. The complete approach. Massachusetts: Blackwell science, Inc.2000; 583-607

2. Hughes J, JS Taylor: Biomechanics and prosthetic practice. In: Murdoch $G$ and Wilson AB Jr, Editors. Amputation surgical practice and patient. Management; 1st edition, Oxford, Butterworth-Heinemann. 1996,41-51

3. Leonard EI, McAnelly R Dm, Lomba M, Faulker VW, Lower Limb Prostheses. Editor Braddom RL, physical medicine and rehabilitation, 2nd ed. Philadelphia; B Saunder; 2000, 279-310

4. Govan NA. Biomechanics and prosthetic management, Editor. G Murdoch and A Bennet Wilson Jr, Amputation surgical practice and patient management; 1996, 59-62

5. Nader Max, Nader Hans George. Otto Bock Prosthetic Compendium- Lower Limb Prostheses, 3rd revised edition, Berlin; Schiele and Schon, 2002, 09-17

6. Zidarov D, Swaine B, Gauthier-Gagnon C. Life habits and prosthetic profile of persons with lower-limb amputation during rehabilitation and at 3-month follow-up. Arch Phys Med Rehabil. 2009 Nov;90 (11):1 953-9. doi: 10.1016/j.apmr. 
2009.06.011

7. 7.Willingham, L. L., Buell, N. C., Allyn, K. J., Hafner, B. J., \& Smith, D.

G. (2004). Measurement of knee center alignment trends in a national sample of established users of the Otto Bock C-Leg microprocessor-controlled knee unit. Journal of Prosthetics and Orthotics, 16(3), 72-75. DOI: 10.1097/00008526200407000-00002

8. Selles RW, Bussmann JBJ, Wagenaar RC, Stam HJ. Effects of prosthetic mass and mass distribution on kinematics and energetics of prosthetic gait: A systematic review. Arch Phys Med Rehabil. December 1999Volume 80, Issue 12, Pages 15931599

9. Gupta PK, Parmar VK, VrC, Mand GS. Patient satisfaction in prosthetic rehabilitation programme. Medical Journal Armed Forces India. April 2001volume 57, issue 2, pages 95-98. doi: http://dx.doi.org/10.1016/s0377-1237(01)80122-2

10. Sahoo J, Mohanty RN, Das SK. Comparative Study of Laminated Exoskeletal versus Modular Endoskeletal Below Knee Prostheses. IJPMR 2010; 21 (1): 5-7 